\title{
Quedas: conceitos, frequências e aplicações à assistência ao idoso. Revisão da literatura
}

\author{
Falls: concepts, frequency and applications to the elderly assistance. Review of the literature
}

\section{Resumo}

Realizou-se revisão da literatura sobre quedas em idosos de comunidade, identificando prevalência, fatores de risco e consequências associadas ao cair, bem como triagem, avaliação e medidas preventivas às quedas na velhice. Foram selecionadas publicações indexadas nas bases de dados Medical Literature Analysis e Retrieval System Online (MEDLINE), por meio do PubMed; Literatura Latino-americana e do Caribe em Ciências da Saúde (LILACS) e Scientific Electronic Library Online (SciELO), relativas ao período de 2008 a 2012, sendo analisadas 41 referências. As evidências levantadas neste estudo destacam as quedas como condição de grande complexidade e que impõe grande desafio para idosos e profissionais da saúde. Ressalta-se a importância da identificação e do manejo dos fatores determinantes e das consequências associadas ao cair, juntamente com o delineamento de estratégias preventivas multifatoriais frente aos idosos vulneráveis ao cair e entre os idosos com história de quedas recorrentes.

\section{Abstract}

A review of the literature on falls in community-dwelling elderly was conducted, identifying prevalence, risk factors and consequences associated to falls, as well as screening, assessment and preventive measures to falls in old age. We selected publications indexed in the following data bases: Medical Literature Analysis and Retrieval System Online (MEDLINE), both consulted through PubMed; (Lilacs) Latin-American and Caribean Science of Health) and Scientific Electronic Library Online (SciELO), from 2008 to 2012; 41 references were analyzed. The evidences surveyed in this study highlight falls as a condition of great complexity that imposes great challenge for elderly and health professionals. We emphasize the importance of identification and management of determinant factors and consequences associated with falls, coupled with the design of multifactorial preventive strategies for elderly vulnerable to falls and among those with history of recurrent falls.

\footnotetext{
Programa de Pós-graduação em Gerontologia, Faculdade de Ciências Médicas. Universidade Estadual de Campinas. Campinas, SP, Brasil.

2 Programa Saúde da Família, Faculdade de Ciências Médicas. Universidade Estadual de Campinas. Campinas, SP, Brasil.
}

Gláucia Regina Falsarella Lívia Pimenta Renó Gasparotto Arlete Maria Valente Coimbra ${ }^{1,2}$

Palavras-chave: Idoso. Acidentes por Quedas. Prevenção.

Key words: Elderly. Accidental Falls. Prevention. 


\section{INTRODUÇÃO}

As quedas apresentam grande importância no cenário populacional, por acometerem um número representativo de idosos. ${ }^{1}$ Em função de sua natureza multifatorial, sua frequência e suas consequências, as quedas constituem uma das grandes síndromes geriátricas e um dos maiores problemas de saúde pública. ${ }^{2-4}$ Além de estarem relacionadas a maior morbidade e mortalidade na velhice, ${ }^{5}$ associam-se a restrição na mobilidade, fraturas, depressão, incapacidade funcional, perda da independência e autonomia, institucionalização e declínio da qualidade de vida, bem como têm gerado implicações socioeconômicas e sobrecarga para os sistemas de saúde. ${ }^{6,7}$

Para a American Geriatrics Society (AGS) e a British Geriatrics Society (BGS), ${ }^{6}$ as quedas são definidas como um contato não intencional com a superfície de apoio, resultante da mudança de posição do indivíduo para um nível inferior à sua posição inicial, sem que tenha havido fator intrínseco determinante ou acidente inevitável e sem perda de consciência. Segundo a Organização Mundial da Saúde (OMS), ${ }^{8}$ esse evento é destacado como causa externa de lesões não intencionais, sendo codificadas pela Classificação International de Doença-9 (CID9) como E880-E888, e pela CID-10 como W00-W19.

No Brasil, o estudo de Coimbra et al. ${ }^{9}$ identificou em 2.209 idosos com idade $\geq 60$ anos e moradores de comunidade uma prevalência de $27,1 \%$ para a ocorrência de quedas e $8,7 \%$ para quedas recorrentes. O levantamento de Siqueira et al., ${ }^{10}$ com 6.616 idosos brasileiros com 65 anos e mais, constatou frequência de $27,6 \%$; de todos os registros de quedas, $11 \%$ resultaram em fratura e $1 \%$ necessitou de atendimento cirúrgico. Na pesquisa de Milat et al., ${ }^{11} 25,6 \%$ da amostra constituída por 5.681 idosos americanos caíram, e destes, $61 \%$ tiveram lesões e 20\% foram hospitalizados. Em âmbito mundial, as quedas são responsáveis por $87 \%$ das fraturas e $50 \%$ das internações nos idosos. ${ }^{8}$

O presente levantamento constitui um resumo da literatura publicada sobre quedas em idosos, especialmente os residentes de comunidade. Estudos sobre o evento multifatorial apresentam grande relevância em âmbito acadêmico, ao aprofundarem o conhecimento sobre este segmento populacional, além de contribuírem para o campo da intervenção, na adoção de estratégias direcionadas à assistência ao idoso nos vários níveis de prevenção e para a redução dos custos em saúde pública. Portanto, este estudo objetivou analisar a prevalência, os fatores de risco, as consequências associadas ao evento, bem como determinar as principais formas de triagem e avaliação e medidas preventivas para as quedas em idosos, por meio de uma revisão da literatura.

\section{MÉTODOS}

Esta revisão sistematizada da literatura investigou estudos sobre quedas em idosos. Foram analisadas publicações indexadas nas bases de dados Medical Literature Analysis e Retrieval System Online (MEDLINE), consultadas por meio do PubMed; Literatura Latino-americana e do Caribe em Ciências da Saúde (LILACS) e Scientific Electronic Library Online (SciELO). A delimitação do período compreendeu os anos de 2008 a 2012. Entretanto, incluem-se três referências consideradas essenciais para o desenvolvimento do estudo: 1) Word Health Organization; 2) American Geriatrics Society (AGS) e British Geriatrics Society (BGS);12 3) Ministério da Saúde ${ }^{13}$ (figura 1). 


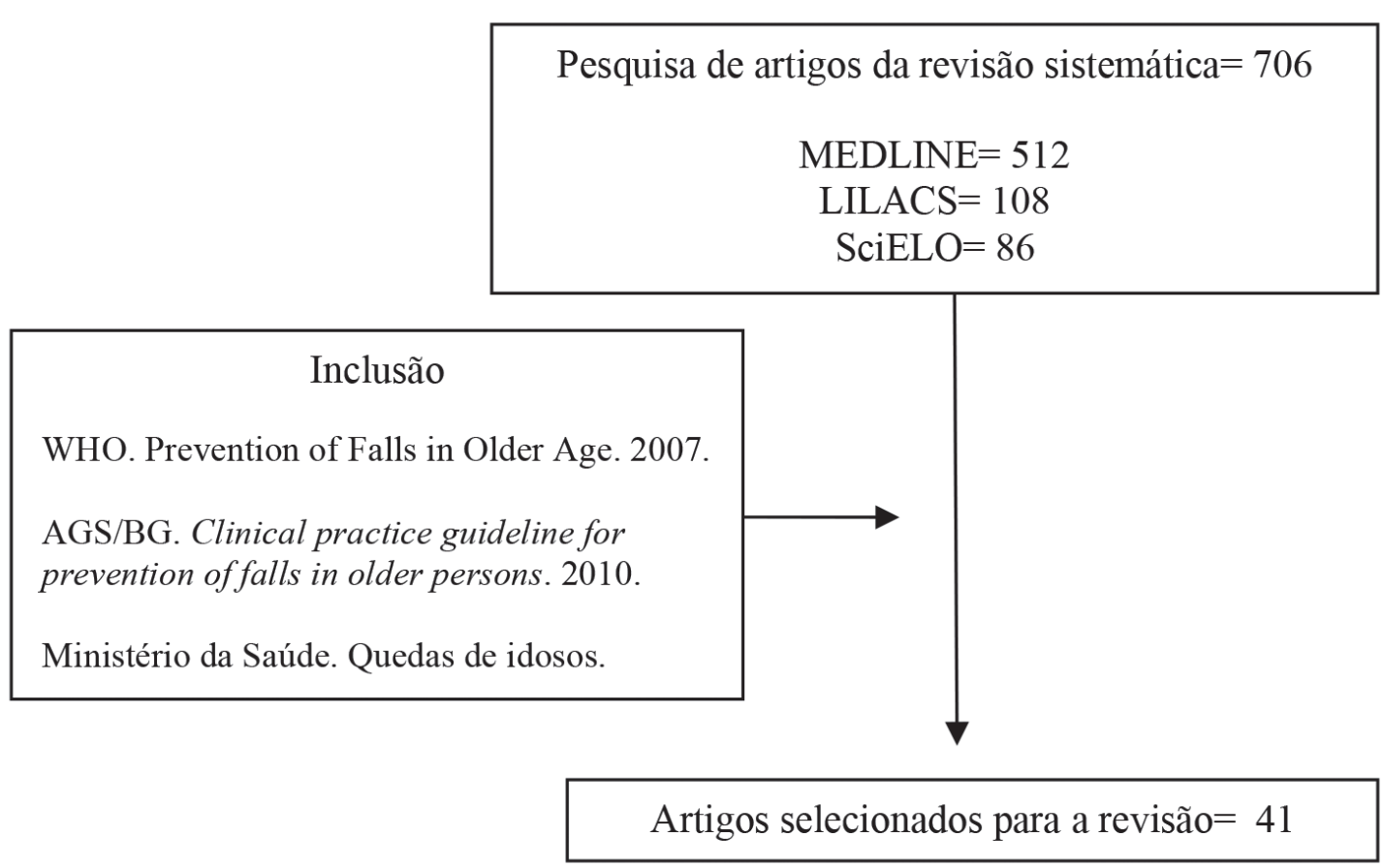

Figura 1. Revisão sistemática de publicações sobre quedas em idosos. 2012.

As buscas foram realizadas definindo-se os seguintes descritores em inglês com os termos "falls and elderly" e "falls and elderly and prevention", para todas as bases de dados pesquisadas. A estratégia para limitação da busca considerou as publicações no idioma em inglês e em português, além de estudos com indivíduos com 60 anos de idade ou mais. A busca foi realizada em outubro de 2012.

Os procedimentos relacionadas a busca, seleção e análise dos artigos foram executados quase que na totalidade do estudo por dois examinadores. Quando necessário, um terceiro examinador era introduzido na investigação para solucionar os casos de discordância em relação à seleção dos estudos.

As 706 referências recuperadas nas buscas foram avaliadas com base nos títulos e resumos, ordenadas por data de publicação, sendo excluídos os artigos que não atendiam aos critérios de inclusão (idosos com 60 anos de idade e mais, dados de prevalência, fatores de risco e/ou avaliação e/ou prevenção de quedas para idosos de comunidade). Em um segundo momento, foi realizada a leitura completa dos artigos selecionados, permitindo identificar critérios de exclusão não observados na etapa anterior e resultando na eliminação de outros estudos (quadro 1). 
Quadro 1. Distribuição de artigos segundo dados de prevalência, fatores associados, consequências e medidas de prevenção para as quedas. 2012.

\begin{tabular}{|c|c|c|c|c|c|c|}
\hline $\begin{array}{l}\text { Autoria, ano e } \\
\text { local do estudo }\end{array}$ & $\begin{array}{l}\text { Tipo de } \\
\text { estudo }\end{array}$ & $\begin{array}{l}\text { Objetivos do } \\
\text { estudo }\end{array}$ & Gênero & Amostra & Idade & Prevalência \\
\hline $\begin{array}{l}\text { Al-Aama, } 2011 \\
\text { Canadá }\end{array}$ & $\begin{array}{c}\text { Revisão } \\
\text { sistematizada }\end{array}$ & $\begin{array}{c}\mathrm{P} * / \mathrm{F}^{* *} / \\
\mathrm{C} \cdot / \mathrm{P} \dagger\end{array}$ & - & - & - & - \\
\hline $\begin{array}{c}\text { Almeida et al., } 2012 \\
\text { Brasil }\end{array}$ & Transversal & $\mathrm{F}^{* *}$ & $\mathrm{M} / \mathrm{F} \neq$ & 267 & $60-90$ & - \\
\hline 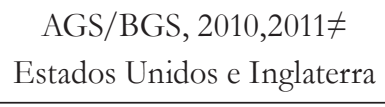 & $\begin{array}{l}\text { Revisão } \\
\text { sistemática }\end{array}$ & $\begin{array}{c}\mathrm{P} * / \mathrm{F}^{* *} / \\
\mathrm{C} \cdot / \mathrm{P} \dagger\end{array}$ & $\mathrm{M} / \mathrm{F} \ddagger$ & - & - & - \\
\hline $\begin{array}{c}\text { Blazer et al., } 2012 \\
\text { Alemanha }\end{array}$ & $\begin{array}{l}\text { Revisão } \\
\text { sistemática }\end{array}$ & $\mathrm{P}+$ & $\mathrm{M} / \mathrm{F} \ddagger$ & - & $\geq 60$ & - \\
\hline $\begin{array}{l}\text { Bleijlevens et al., } 2010 \\
\text { Holanda }\end{array}$ & Transversal & $\mathrm{C} \bullet$ & & 333 & $\geq 65$ & \\
\hline $\begin{array}{l}\text { Choi et al., } 2012 \\
\text { Estados Unidos }\end{array}$ & $\begin{array}{c}\text { Revisão } \\
\text { sistemática/ } \\
\text { Meta-análise }\end{array}$ & $\mathrm{P} \dagger$ & - & - & - & - \\
\hline $\begin{array}{l}\text { Ciaschini et al., } 2009 \\
\text { Canadá }\end{array}$ & Longitidinal & $\mathrm{P} * / \mathrm{C} \bullet$ & $\mathrm{M} / \mathrm{F} \ddagger$ & 201 & $\geq 55$ & $41 \%$ \\
\hline $\begin{array}{c}\text { Coimbra et al., } 2010 \\
\text { Brasil }\end{array}$ & Transversal & $\begin{array}{c}\mathrm{P} * / \mathrm{F} * * / \\
\mathrm{C}^{* * *}\end{array}$ & $\mathrm{M} / \mathrm{F} \ddagger$ & 2.209 & $\geq 60$ & $27 \%$ \\
\hline $\begin{array}{c}\text { Coutinho et al., } 2009 \\
\text { Brasil }\end{array}$ & Caso-controle & $\mathrm{C}^{* * *}$ & $\mathrm{M} / \mathrm{F} \ddagger$ & 414 & $\geq 60$ & - \\
\hline $\begin{array}{c}\text { Cruz et al., } 2012 \\
\text { Brasil }\end{array}$ & Transversal & $\mathrm{P} * / \mathrm{F}^{* *}$ & $\mathrm{M} / \mathrm{F} \ddagger$ & 420 & $\geq 60$ & $32 \%$ \\
\hline $\begin{array}{c}\text { Czerwinski et al., } 2008 \\
\text { Polônia }\end{array}$ & Revisão & $\begin{array}{c}\mathrm{P} * / \mathrm{F}^{* *} / \\
\mathrm{C} \cdot / \mathrm{P} \dagger\end{array}$ & $\mathrm{M} / \mathrm{F} \ddagger$ & - & - & $30-40 \%$ \\
\hline $\begin{array}{c}\text { Deandrea et al., } 2010 \\
\text { Itália }\end{array}$ & $\begin{array}{c}\text { Revisão } \\
\text { sistemática/ } \\
\text { Meta-análise }\end{array}$ & $\mathrm{F}^{* *}$ & $\mathrm{M} / \mathrm{F} \ddagger$ & - & - & - \\
\hline $\begin{array}{l}\text { Dias et al., } 2011 \\
\text { Brasil }\end{array}$ & Transversal & Medo cair & $\mathrm{M} / \mathrm{F} \ddagger$ & 113 & $\geq 65$ & - \\
\hline $\begin{array}{c}\text { Elsawy et al., } 2011 \\
\text { Estados Unidos }\end{array}$ & $\begin{array}{l}\text { Revisão } \\
\text { sistemática }\end{array}$ & $\begin{array}{l}\text { Avaliação } \\
\text { Geriátrica }\end{array}$ & - & - & - & - \\
\hline $\begin{array}{l}\text { Fabre et al., } 2010 \\
\text { Estados Unidos }\end{array}$ & $\begin{array}{l}\text { Revisão } \\
\text { integrativa }\end{array}$ & $\mathrm{F}^{* *}$ & - & - & - & - \\
\hline $\begin{array}{l}\text { Finlayson et al., } 2010 \\
\text { Estados Unidos }\end{array}$ & $\begin{array}{c}\text { Revisão } \\
\text { sistematizada }\end{array}$ & $\begin{array}{c}\mathrm{P} * / \mathrm{F}^{* *} / \\
\mathrm{C} \cdot / \mathrm{P} \dagger\end{array}$ & $\mathrm{M} / \mathrm{F} \ddagger$ & - & $\begin{array}{l}\geq 65 \\
\geq 75\end{array}$ & $\begin{array}{l}30 \% \\
50 \%\end{array}$ \\
\hline $\begin{array}{l}\text { Fox et al., } 2010 \\
\text { Estados Unidos }\end{array}$ & $\begin{array}{l}\text { Ensaio } \\
\text { clínico }\end{array}$ & $\mathrm{P} \dagger$ & $\mathrm{M} / \mathrm{F} \ddagger$ & 543 & $\geq 65$ & - \\
\hline $\begin{array}{c}\text { Freiberger et al., } 2012 \\
\text { Alemanha }\end{array}$ & $\begin{array}{l}\text { Ensaio } \\
\text { clínico }\end{array}$ & $\mathrm{P} \dagger$ & $\mathrm{M} / \mathrm{F} \neq$ & 280 & $70-90$ & - \\
\hline $\begin{array}{c}\text { Galizia et al., } 2012 \\
\text { Itália }\end{array}$ & Longitudinal & $\begin{array}{c}\mathrm{P} * / \mathrm{F}^{* *} / \\
\mathrm{C} \cdot\end{array}$ & $\mathrm{M} / \mathrm{F} \neq$ & 1.290 & $\geq 65$ & $13,8 \%$ \\
\hline
\end{tabular}




\begin{tabular}{|c|c|c|c|c|c|c|}
\hline $\begin{array}{c}\text { Gates et al., } 2008 \\
\text { Inglaterra }\end{array}$ & $\begin{array}{c}\text { Revisão / } \\
\text { Meta-análise }\end{array}$ & $\mathrm{F}^{* *} / \mathrm{P} \dagger$ & $\mathrm{M} / \mathrm{F} \ddagger$ & - & $\geq 60$ & - \\
\hline $\begin{array}{c}\text { Gschwinda et al., } 2011 \\
\text { Suíça }\end{array}$ & $\begin{array}{l}\text { Revisão } \\
\text { sistemática }\end{array}$ & $\mathrm{P} \dagger$ & $\mathrm{M} / \mathrm{F} \ddagger$ & - & $\geq 60$ & - \\
\hline $\begin{array}{c}\text { Kwan et al., } 2011 \\
\text { Austrália }\end{array}$ & $\begin{array}{c}\text { Revisão } \\
\text { sistemática }\end{array}$ & $\begin{array}{c}\mathrm{P} * / \mathrm{F}^{* *} / \\
\mathrm{C} \cdot\end{array}$ & $\mathrm{M} / \mathrm{F} \ddagger$ & - & & $14,7-37 \%$ \\
\hline $\begin{array}{l}\text { Michael et al., } 2010 \\
\text { Estados Unidos }\end{array}$ & $\begin{array}{l}\text { Revisão } \\
\text { sistemática }\end{array}$ & $\mathrm{P} \dagger$ & $\mathrm{M} / \mathrm{F} \ddagger$ & - & $\geq 60$ & - \\
\hline $\begin{array}{c}\text { Milat et al., } 2011 \\
\text { Austrália }\end{array}$ & Transversal & $\begin{array}{c}\mathrm{P} * / \mathrm{F}^{* *} / \\
\mathrm{C} \bullet\end{array}$ & $\mathrm{M} / \mathrm{F} \ddagger$ & 5.681 & $\geq 65$ & $25,6 \%$ \\
\hline $\begin{array}{c}\text { MS, 2011*** } \\
\text { Brasil }\end{array}$ & Longitudinal & $\mathrm{C} \bullet$ & - & - & - & - \\
\hline $\begin{array}{l}\text { Morley et al., } 2012 \\
\text { Estados Unidos }\end{array}$ & Editorial & $\mathrm{C} \bullet$ & - & - & & \\
\hline $\begin{array}{c}\text { Nicolussi et al., } 2012 \\
\text { Brasil }\end{array}$ & $\begin{array}{c}\text { Revisão } \\
\text { integrativa }\end{array}$ & $\mathrm{F}^{* *} / \mathrm{C} \bullet$ & - & - & $\geq 60$ & - \\
\hline $\begin{array}{c}\text { WHO, 2010" } \\
\text { Brasil }\end{array}$ & $\begin{array}{c}\text { Revisão } \\
\text { sistematizada }\end{array}$ & $\begin{array}{c}\mathrm{P} * / \mathrm{F}^{* *} / \\
\mathrm{C} \cdot / \mathrm{P} \dagger\end{array}$ & $\mathrm{M} / \mathrm{F} \ddagger$ & - & $\begin{array}{l}\geq 60 \\
\geq 70\end{array}$ & $\begin{array}{l}28-35 \% \\
32-42 \%\end{array}$ \\
\hline $\begin{array}{c}\text { Parreira et al., } 2010 \\
\text { Brasil }\end{array}$ & Transversal & $\begin{array}{c}\mathrm{P}^{*} / \mathrm{F}^{* *} / \\
\mathrm{C} \bullet\end{array}$ & $\mathrm{M} / \mathrm{F} \ddagger$ & 1.993 & $\geq 60$ & $32,1 \%$ \\
\hline $\begin{array}{c}\text { Peeters et al., } 2009 \\
\text { Holanda }\end{array}$ & $\begin{array}{c}\text { Revisão } \\
\text { sistemática }\end{array}$ & $\begin{array}{c}\mathrm{P}^{*} / \mathrm{F}^{* *} / \\
\mathrm{C} \bullet\end{array}$ & $\mathrm{M} / \mathrm{F} \ddagger$ & - & $\geq 60$ & $30 \%$ \\
\hline $\begin{array}{c}\text { Ricci et al., } 2009 \\
\text { Brasil }\end{array}$ & Transversal & $\mathrm{F}^{* *}$ & $\mathrm{M} / \mathrm{F} \ddagger$ & 96 & $\geq 60$ & - \\
\hline $\begin{array}{l}\text { Scheffer et al., } 2008 \\
\text { Holanda }\end{array}$ & $\begin{array}{c}\text { Revisão } \\
\text { sistemática }\end{array}$ & Medo de cair & $\mathrm{M} / \mathrm{F} \ddagger$ & - & $\geq 65$ & - \\
\hline $\begin{array}{l}\text { Scott et al., } 2011 \\
\text { Canadá }\end{array}$ & $\begin{array}{l}\text { Revisão } \\
\text { sistemática/ } \\
\text { Longitudinal }\end{array}$ & $\mathrm{P} \dagger$ & - & - & - & - \\
\hline $\begin{array}{l}\text { Shin et al., } 2011 \\
\text { Coreia }\end{array}$ & Transversal & $\mathrm{P} * / \mathrm{F}^{* *}$ & $\mathrm{M} / \mathrm{F} \ddagger$ & 110 & $\geq 65$ & $48,1 \%$ \\
\hline $\begin{array}{c}\text { Siqueira et al., } 2011 \\
\text { Brasil }\end{array}$ & Transversal & $\mathrm{P} * / \mathrm{F}^{* *}$ & $\mathrm{M} / \mathrm{F} \ddagger$ & 6.616 & $\geq 60$ & $27,6 \%$ \\
\hline $\begin{array}{l}\text { Stevens et al., } 2010 \\
\text { Estados Unidos }\end{array}$ & $\begin{array}{c}\text { Revisão } \\
\text { sistematizada }\end{array}$ & $\begin{array}{c}\mathrm{P} * / \mathrm{F}^{* *} / \\
\mathrm{C} \cdot / \mathrm{P} \dagger\end{array}$ & $\mathrm{M} / \mathrm{F} \ddagger$ & - & $\geq 65$ & $30 \%$ \\
\hline $\begin{array}{l}\text { Tinetti et al., } 2010 \\
\text { Estados unidos }\end{array}$ & $\begin{array}{l}\text { Estudo de } \\
\text { caso }\end{array}$ & $\begin{array}{c}\mathrm{P} * / \mathrm{F}^{* *} / \\
\mathrm{C} \cdot / \mathrm{P} \dagger\end{array}$ & $\mathrm{F} \ddagger$ & 1 & 89 & - \\
\hline $\begin{array}{l}\text { Wang et al., } 2010 \\
\text { China }\end{array}$ & $\begin{array}{c}\text { Revisão } \\
\text { sistematizada }\end{array}$ & $\begin{array}{c}\mathrm{P} * / \mathrm{F}^{* *} / \\
\mathrm{C} \cdot / \mathrm{P} \dagger\end{array}$ & $\mathrm{M} / \mathrm{F} \ddagger$ & - & $\geq 60$ & $18 \%$ \\
\hline $\begin{array}{c}\text { WHO, } 2007 \\
\text { Canadá }\end{array}$ & $\begin{array}{c}\text { Revisão } \\
\text { sistematizada }\end{array}$ & $\mathrm{P} \dagger$ & & & & \\
\hline \multicolumn{7}{|c|}{$\begin{array}{l}* \mathrm{P}=\text { prevalência de quedas; } * * \mathrm{~F}=\text { fatores associados às quedas; } \cdot \mathrm{C}=\text { consequências associadas às quedas; } \nmid \mathrm{P}=\text { prevenção para as quedas; } \ddagger \mathrm{M} \\
\mathrm{F}=\text { masculino e feminino; } * * * \mathrm{MS}=\text { Ministério da Saúde; } \cdot \mathrm{WHO}=\text { World Health Organization; } \neq \mathrm{AGS} \text { e BGS,2010= American Geriatrics Societ } \\
\text { and British Geriatrics Society. }\end{array}$} \\
\hline
\end{tabular}


Para a avaliação da qualidade metodológica dos artigos, considerou-se a classificação de trabalhos científicos ${ }^{14}$ com base no desenho empregado na geração de evidência: nível A
- revisão sistemática e ensaios randomizados; nível B - estudos de coorte e prospectivos; nível C - estudos retrospectivos; nível D - opinião de especialista e decisão de consenso (quadro 2).

Quadro 2. Avaliação da qualidade metodológica dos artigos baseada na geração de evidência. 2012.

\begin{tabular}{|c|c|c|c|c|}
\hline Nível A & Nível B & Nível C & Nível D & Sem classificação* \\
\hline $\begin{array}{c}11 \text { revisões sistemáticas } \\
2 \text { ensaios clínicos }\end{array}$ & 3 longitudinais & 1 retrospectivo & 2 consensos & $\begin{array}{c}10 \text { transversais } \\
6 \text { revisões sistematizadas } \\
2 \text { revisões integrativas } \\
1 \text { revisão }\end{array}$ \\
\hline
\end{tabular}

*artigos que não se enquadraram nas quatro classificações.

\section{RESULTADOS E DISCUSSÃO}

\section{Prevalência e consequências associadas às quedas}

Mais de um terço dos idosos de comunidade com 65 anos de idade e mais caem anualmente, e esta proporção aumenta para $32-42 \%$ entre aqueles com mais de 70 anos, atingindo $50 \%$ dos idosos com 80 anos ou mais $;{ }^{15} \mathrm{em}$ metade desses casos, as quedas são recorrentes. ${ }^{16} \mathrm{~A}$ ocorrência deste evento se amplia com o avanço da idade e o nível de fragilidade; assim, os idosos institucionalizados têm maior risco de cair, se comparados com aqueles residentes de comunidade. ${ }^{17}$ De 30 a $50 \%$ das pessoas com 65 anos e mais e que vivem em instituições de longa permanência para idosos caem a cada ano. ${ }^{8}$ As mulheres sofrem três vezes mais quedas, quando comparadas aos homens, mas no geral a mortalidade associada a esta condição é maior no gênero masculino. ${ }^{18}$

Cair constitui fator desencadeador de lesões de tecidos moles, lacerações e fraturas. ${ }^{19,20}$ Mais de $30 \%$ dos idosos que caem passam a ter declínio em sua funcionalidade. Aproximadamente $5 \%$ de todas as quedas em idosos da comunidade resultam em fratura, ${ }^{4}$ e de $5 \%$ a $10 \%$, em grave lesão de tecido mole e de cabeça. ${ }^{21}$ As quedas associamse ainda a $12 \%$ das mortes no mundo entre os idosos, representando $40 \%$ dos óbitos por lesões nesta faixa etária, sendo a quinta causa de morte na velhice ${ }^{18}$ e a primeira por causa externa. ${ }^{22}$ Após as quedas, $20 \%$ dos idosos morrem dentro de um ano, como consequência da fratura de quadril. ${ }^{23}$
Além disso, cair pode resultar em uma síndrome pós-queda que inclui dependência, perda de autonomia, imobilização, isolamento e depressão, o que levará a uma maior restrição nas tarefas diárias. ${ }^{23}$ A literatura, porém, descreve que independentemente de terem sofrido queda, os idosos em geral têm baixo senso de eficácia para evitar o episódio, apresentam medo de cair, restrição em atividades, perda de confiança em si mesmos $^{24}$ e pior qualidade de vida. ${ }^{17}$

A síndrome pós-queda e o medo de cair afetam $73 \%$ daqueles que sofreram o evento no ano anterior; já entre os idosos sem relato de quedas recentes, a prevalência foi de $46 \%{ }^{25}$ As quedas podem provocar um ciclo vicioso, o qual leva a redução da capacidade funcional e, por conseguinte, maior suscetibilidade do idoso sofrer novas quedas.

O impacto econômico atribuído às quedas e as fraturas varia muito entre os países, em função das diferentes políticas de saúde de cada localidade e da diversidade de condições socioeconômicas das populações. ${ }^{26}$ No Brasil, a cada ano, o SUS tem gastos crescentes com medicamentos, consultas médicas, tratamentos e reabilitações para o grupo etário que sofreu quedas. Em 2009, foram $\mathrm{R} \$ 57,61$ milhões com internações, $\mathrm{R} \$ 24,77$ milhões com medicamentos para o tratamento de osteoporose e quase $\mathrm{R} \$ 81$ milhões com as fraturas em idosos. ${ }^{13}$ Nos Estados Unidos, em 2000, o custo financeiro com tratamento de quedas na velhice ultrapassou 
U\$19 bilhões, e para 2020 as projeções apontam para um encargo de U $\$ 55$ bilhões. ${ }^{25}$

\section{Fatores associados às quedas}

Evidências na literatura apontam para a existência de aproximadamente 400 diferentes fatores de risco para as quedas. ${ }^{18}$ Subjacentes a uma queda encontram-se a associação entre as disfunções de múltiplos sistemas e órgãos e a influência de aspectos externos ao indivíduo. ${ }^{27}$ Ao menos duas condições de saúde simultâneas e um fator ambiental desempenham papel decisivo na maior parte das ocorrências entre os idosos. No entanto, em cerca de $8 \%$ dos casos nenhum fator de risco é determinado. ${ }^{18}$
Este evento multifatorial envolve aspectos intrínsecos e extrínsecos, ${ }^{28,29}$ e contempla as dimensões biológica, comportamental, ambiental e socioeconômica (figura 2). ${ }^{30}$ Os estudos têm mostrado clara tendência para os preditores de quedas na velhice, como idade avançada, gênero feminino, incapacidade funcional, história pregressa de quedas, distúrbios de marcha e de equilíbrio, baixa aptidão física, baixo índice de massa corporal, diminuição da força muscular, hipotensão postural, tontura, alteração cognitiva, depressão, doença cerebrovascular e neurológica, incontinência urinária, declínio da acuidade visual, problemas nos pés, risco ambiental e uso concomitante de vários fármacos. ${ }^{10,12,31}$

\section{Fatores de risco biológico}

- Idade avançada

- Quedas anteriores

- Sexo feminino

- Fraqueza muscular

- Falta de equilíbrio e coordenação

- Distúrbios da marcha

- Condições crônicas (artrite diabetes, AVC, Parkinson, incontinência, demência problemas de visão)

- Limitações funcionais

- Doenças aguda

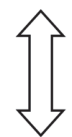

Fatores de risco ambiental

- Falta de corrimãos em escada

- Má concepção escada

- Falta de barras de apoio em banheiro

- Pouca iluminação ou brilho

- Obstáculos para tropeço

- Superfícies escorregadias ou irregulares

- Espaços públicos ou construções mal projetados e/ou mantidos

\section{Fatores de risco comportamental}

- Uso de vários medicamentos

- Inatividade física

- Medo de cair

- Uso inadequado de auxiliares de locomoção

- Má nutrição ou hidratação

- Calçado impróprio

- Uso de álcool

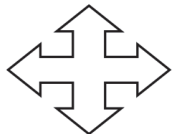

Fatores de risco socioeconômico

- Baixa renda

- Falta de educação

- Más condições de vida

- Falta de apoio social

- Viver sozinho

- Barreiras de analfabetismo

\section{Quedas e lesões relacionadas às quedas}

Figura 2. Modelo conceitual dos fatores de risco para quedas em idosos.

Adaptado $\mathrm{OMS}^{23}$ e Stevens et al. ${ }^{30}$ 
Em estudo realizado por Coimbra et al., ${ }^{9}$ as variáveis relacionadas ao cair foram idade maior ou igual a 80 anos, gênero feminino, presença de oito ou mais doenças, necessidade de hospitalizações, presença de sintomas depressivos, problemas de audição e baixa qualidade de vida para o aspecto emocional. Outra pesquisa nacional, com 113 idosos comunitários, investigou história de quedas, restrição de atividades por medo de cair, autoeficácia relacionada a quedas, fenótipo de fragilidade, aspectos sociodemográficos e clínicos, capacidade funcional, depressão e autopercepção de saúde. Segundo os resultados, os idosos com restrição de atividades por medo de cair relataram menor autoeficácia para quedas, maior depressão, menor velocidade de marcha e dependência para as atividades instrumentais de vida diária, maior número de doenças e pior autopercepção de saúde. ${ }^{32}$

Tão importante quanto identificar os preditores de quedas, é valorizar a interação entre os múltiplos fatores, uma vez que a ocorrência desse evento aumenta com o número de variáveis associadas. Segundo a American Geriatrics Society e a British Geriatrics Society, ${ }^{6}$ o percentual de idosos residentes em comunidade que sofreram quedas aumentou de $27 \%$ para aqueles com até um fator de risco, e para $78 \%$ entre aqueles com quatro ou mais fatores associados. Verificou-se também que a frequência de idosos de comunidade com quedas recorrentes aumentou de $10 \%$ para $69 \%$ quando o número de fatores de risco aumentou de um para quatro ou mais.

Nesse contexto, Tinetti \& $\mathrm{Kumar}^{33}$ relatam que em um ano o risco de cair dobra para cada fator adicional. Assim, a chance de um idoso cair é de $8 \%$ quando não exposto a fatores de risco, chegando a 78\% na presença de quatro variáveis. De acordo com Deandrea et al..$^{34}$ e Al-Aama, ${ }^{16}$ os fatores de risco com associação mais forte para as quedas foram história de quedas, problemas de marcha, uso de dispositivo de auxílio para caminhar, vertigem, doença de Parkinson e uso de antiepiléptico.

Em geral, as quedas ocasionais podem ser atribuídas principalmente a fatores extrínsecos, enquanto as recorrentes são causadas por aspectos intrínsecos, acompanhados de risco ambiental. As quedas recorrentes são definidas como dois ou mais episódios dentro de um período de seis meses. A causalidade das quedas é complexa, à medida que muitos fatores de risco atuam simultaneamente. No entanto, o risco atribuível de cada um desses fatores preditores é limitado, sendo que o indicador mais importante é a história pregressa do cair. Assim, em idosos de comunidade o risco de cair é duas vezes maior em pessoas que já sofreram uma queda anteriormente. ${ }^{26}$

Aproximadamente $55 \%$ das quedas acontecem no ambiente ao ar livre e apenas 20\% no período da noite. ${ }^{23}$ Já o estudo de Coutinho et al. ${ }^{19}$ evidenciou que a maioria das quedas foi registrada no domicílio, entre 6 e 18 horas, mas, as circunstâncias das quedas seguidas por fraturas podem variar segundo gênero e idade. Essa diversidade de achados relativos à ocorrência de quedas está provavelmente associada à heterogeneidade das amostras de idosos e dos instrumentos metodológicos utilizados nos estudos mencionados.

As limitações na mobilidade representa uma das principais razões de quedas no ambiente interno; evidências na literatura apontam que $50 \%$ dos casos são precedidos por escorregões ou tropeções; 20-30\% estão relacionados a alterações no equilíbrio; $10 \%$ associam-se a vertigem; e $10 \%$ são decorrentes de desmaios. ${ }^{23}$

Fatores neurológicos que predispõem às quedas referem-se a alterações da função vestibular, proprioceptiva e cognitiva. A alteração no equilíbrio pode estar relacionada à insuficiência dos mecanismos neurais e osteoarticulares envolvidos na manutenção da postura corporal. $\mathrm{O}$ mecanismo sensorial assume papel fundamental no equilíbrio, e a integração das informações visuais, proprioceptivas e vestibulares é necessária para gerar respostas apropriadas para a manutenção do controle postural. ${ }^{35}$

Assistência ao idoso no evento da queda: triagem e avaliação

O questionamento sobre a manifestação de quedas constitui parte do interrogatório 
complementar anual da consulta clínica, para identificação de sinais e sintomas apresentados durante o episódio. Atenção especial será destinada às circunstâncias, como hora, dia da semana, local e descrição da atividade exercida no momento da queda, juntamente com os fatores de risco. A revisão da medicação é de grande importância, bem como as investigações funcionais e ambientais.

Quando a queda está associada com lesão ou relacionada à perda de consciência, febre, alteração da pressão arterial ou história de quedas recorrentes, é necessária uma avaliação emergencial, uma vez que em alguns casos é possível verificar fatores que contribuíram para o cair, como a manifestação não específica de muitas doenças agudas ou sinal de descompensação de processos crônicos. A identificação de alterações cognitivas no indivíduo idoso pode excluir possíveis condições, como hipotensão postural, arritmias, anemia, desidratação, efeitos colaterais de algumas medicações, síncope, acidente vascular transitório e doenças do labirinto.

Com base na literatura investigada nesta revisão, e mais especificamente nos consensos de AGS/BGS ${ }^{12}$ e $\mathrm{WHO}^{8}{ }^{8}$ segue sugestão de triagem e avaliação anual direcionadas aos profissionais de saúde que prestam assistência aos idosos: 1) questionar os idosos se eles caíram no último ano; 2) para aqueles que caíram, perguntar sobre a frequência e as circunstâncias; 3) detectar dificuldades na mobilidade ou no equilíbrio; 4) para os idosos com quedas no ano anterior ou com dificuldades no caminhar ou no equilíbrio, torna-se essencial uma avaliação multifatorial do risco de queda; 5) para aqueles que caíram, é fundamental avaliar a marcha e o equilíbrio; 6) a idosos que não conseguem realizar ou desempenham de forma insatisfatória o teste de marcha e de equilíbrio, recomenda-se avaliar o risco multifatorial; 7) avaliação de risco multifatorial de queda deve ser executada por um médico com habilidades e formação adequada.

Segundo a AGS/BGS, ${ }^{12}$ os idosos que caíram apenas uma vez no ano anterior e não necessitaram de atendimento médico deverão passar primeiro por avaliação do equilíbrio e marcha - apenas se apresentarem alteração nestes, deverão ser encaminhados para avaliação multifatorial.

Por se tratar de evento de etiologia multifatorial, há dificuldade no estabelecimento de instrumentos de rastreio para o risco de quedas. Assim, a identificação de idosos vulneráveis ou com alto risco para cair necessita de avaliação multidimensional. ${ }^{36}$ A avaliação geriátrica ou ampla permite identificar as incapacidades e limitações, como a necessidade de cuidados, direcionando a intervenção. Esse tipo de avaliação enfatiza a funcionalidade da pessoa idosa. ${ }^{37} \mathrm{Na}$ investigação do risco multifatorial para quedas, é essencial incluir a história de saúde do indivíduo conforme descrito anteriormente, bem como avaliação da função física, análise do componente sensorial (audição e visão), medida de sinais ortostáticos e neurológico, avaliação musculoesquelética, disfunção cognitiva e depressão. ${ }^{25}$

Em relação à seleção de ferramentas de rastreio, é de extrema importância a verificação da validade do instrumento junto à população idosa, além da compreensão das limitações impostas pelas ferramentas. Outro fator relevante se refere à interpretação correta dos resultados obtidos nos testes, a fim de identificar as incapacidades e limitações, assim como as necessidades de cuidados, para o direcionamento de intervenções eficazes. ${ }^{25}$

\section{Prevenção de quedas}

A estratégia para orientação quanto à prevenção de quedas recomenda que o médico da atenção primária seja o profissional indicado para avaliar anualmente, em seus pacientes idosos, os fatores de risco que podem ocasionar esse evento multifatorial, bem como implementar medidas preventivas. Assim, o algoritmo clínico descreve o processo sistemático de tomada de decisão e de intervenção junto aos idosos com quedas ou com dificuldade de caminhar ou de equilíbrio ${ }^{6}$ (figura 3). 


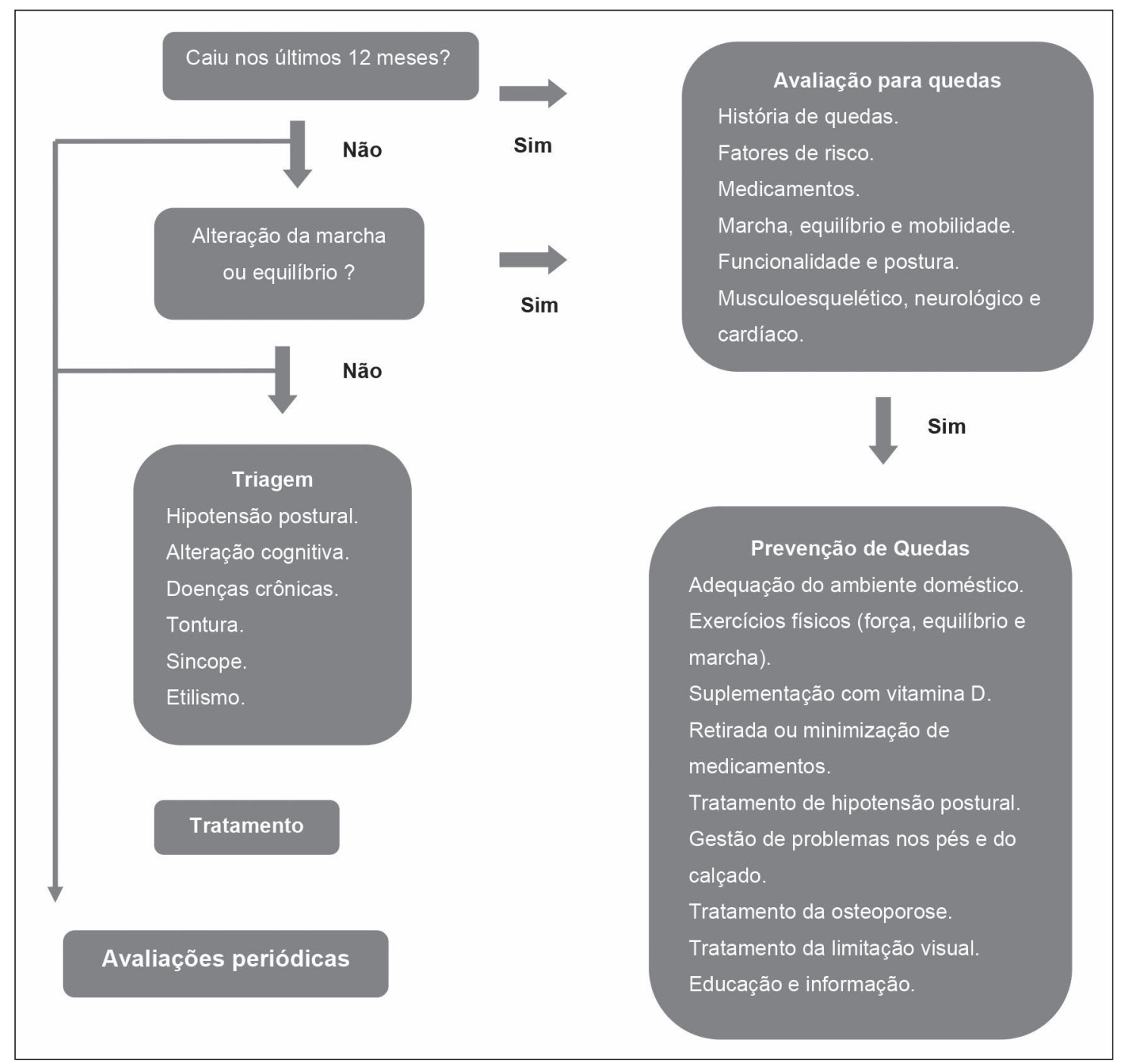

Figura 3. Abordagem sugerida para avaliação e prevenção de quedas em idosos.

Adaptado Al-Aama. ${ }^{16}$

Levantamentos evidenciam a existência de vários tipos de intervenção para a prevenção de quedas em idosos, ${ }^{38}$ que são em geral divididas em intervenção única ou multicomponente. ${ }^{16}$ De maneira mais específica, as diretrizes pautadas tanto pela $\mathrm{AGS} / \mathrm{BGS}^{12}$ quanto pela $\mathrm{WHO}^{8}$ destacam os elementos mais comumente incluídos em intervenções eficazes, segundo o nível de evidência de efetividade das ações (quadro 3). 
Quadro 3. Intervenções para prevenção de quedas em idosos, segundo o nível de evidência de efetividade das ações. 2012.

\section{Nível de evidência A}

Intervenções com forte evidência na literatura e com resultados importantes para a saúde, as quais deverão ser recomendadas pelos médicos aos pacientes elegíveis:

- adaptação ou modificação do ambiente doméstico é indicada aos idosos que caíram ou que apresentem fatores de risco;

- incorporação de um programa de exercícios físicos, em específico de força, equilíbrio e marcha. Os treinamentos de flexibilidade e de resistência devem ser incluídos, mas não como elementos únicos do programa;

- suplementos de vitamina D para idosos com deficiência e com dosagem de pelo menos 800UI por dia;

- intervenção realizada por profissionais da área da saúde, por intermédio da avaliação do risco de cair, juntamente com a implementação de medidas necessárias.

\section{Nível de evidência B}

Intervenções que apresentam, pelo menos, um mínimo de evidências de resultados positivos à saúde e, portanto, os médicos podem propor estas ações aos idosos elegíveis:

- retirada ou redução da dosagem de medicamentos psicoativos;

- redução das doses ou do número de outros medicamentos;

- avaliação da hipotensão postural.

\section{Nível de evidência $\mathrm{C}$}

Intervenções com nenhuma recomendação a favor ou contra são feitas. Pelo menos um mínimo de evidência foi observado com relação aos resultados de melhora da saúde, porém os benefícios e prejuízos são muito próximos para promover uma recomendação geral:

- identificação e tratamento para problemas nos pés (joanetes, úlceras ou unhas deformadas). Atenção dada aos calçados é importante, além de evitar o uso de sapatos gastos ou com saltos;

- programas de educação e informação sobre quedas.

Fontes: $\mathrm{WHO}^{8}$; AGS/BGS. ${ }^{12}$

Várias pesquisas baseadas em ensaios clínicos randomizados e estudos quase-experimentais apresentam conclusões conflitantes a respeito da efetividade dos programas de intervenção para prevenção de quedas. A avaliação da eficácia clínica dessas intervenções é dificultada por problemas metodológicos e pela heterogeneidade das pesquisas clínicas - amostra do estudo, tipo de intervenção, controle e avaliação da intervenção e qualidade metodológica dos estudos. ${ }^{36,39,40}$ Uma meta-análise investigou ensaios clínicos randomizados publicados nos últimos dez anos sobre a efetividade das intervenções para prevenção de quedas, e segundo os resultados do estudo, as intervenções se mostraram modestamente eficazes. ${ }^{41}$

No entanto, a intervenção multifatorial tem sido sugerida pelos trabalhos acadêmicos como a mais eficaz, quando comparada com a intervenção única. Além disso, a AGS/BGS 6 recomenda as intervenções multicomponentes como estratégia de tratamento primário na orientação para a prevenção de quedas. 
Entre as limitações deste estudo, menciona-se a execução de uma revisão sistematizada e não sistemática da literatura. Em relação à qualidade metodológica dos estudos analisados, constatase que metade das investigações não possuía critérios para serem classificadas segundo o nível de evidência, sugerindo, portanto, maior rigor no processo de elaboração de futuros levantamentos.

\section{CONCLUSÃO}

As evidências levantadas neste estudo destacam as quedas como uma das síndromes geriátricas e grande problema de saúde pública na atualidade. Sua prevalência apresentou

\section{REFERÊNCIAS}

1. Scott V, Gallagher E, Higginson A, Metcalfe S, Rajabali F. Evaluation of an evidence-based education program for health professionals: the Canadian Falls Prevention Curriculum(C) (CFPC). J Safety Res 2011;42(6):501-7.

2. Cruz DT, Ribeiro LC, Vieira MT, Teixeira MTB, Bastos RB, Leite ICG. Prevalence of falls and associated factors in elderly individuals. Rev Saúde Pública 2012;46(1):138-46.

3. Shim EY, Ma SH, Hong SH, Lee SN, Paik WY, Seo DS et al. Correlation between frailty level and adverse healthrelated outcomes of communitydwelling elderly, one year retrospective study. Korean J Fam Med 2011;32(4):249-56.

4. Wang J, Chen Z, Song Y. Falls in aged people of the Chinese mainland: epidemiology, risk factors and clinical strategies. Ageing Res Rev 2010;9 Suppl: S13-17.

5. Parreira JG, Vianna AMF, Cardoso GS, Karakhanian WZ, Calil D, Perlingeiro JAG et al. Lesões graves em vítimas de queda da própria altura. Rev Assoc Med Bras 2010;56(6):660-4.

6. American Geriatrics Society; British Geriatrics Society. AGS/BGS Clinical practice guideline: for prevention of falls in older persons [Internet]. New York: AGS; 2010 [acesso em 20 ago 2012]. Disponível em: http://www.americangeriatrics.org/ health_care_professionals/clini cal_practice/clinical_ guidelines_recommendations/2010/ variação entre os estudos, mas a frequência esteve elevada. Entre os principais fatores associados a este evento mutifatorial, destacamse aqueles relacionados às dimensões biológica, comportamental, ambiental e socioeconômica. Com relação ao desfecho das quedas, os estudos sugerem incapacidades, fraturas, internações e mortalidade. Quanto às medidas de prevenção para as quedas, a intervenção multifatorial é descrita pelos trabalhos acadêmicos como a mais eficaz, utilizando adaptação do ambiente, incorporação de exercícios físicos, suplementação com vitamina $\mathrm{D}$, retirada ou redução da dosagem de medicamentos, em especial os psicoativos, e avaliação da hipotensão postural.

7. Finlayson ML, Peterson EW. Falls, aging and disability. Phys Med Rehabil Clin North Am 2010;21(2): 357-73.

8. Scott V. World Heath Organization Report: prevention of Falls in Older Age. [Internet]. Geneva: WHO; 2007 [acesso em 16 Out 2012]. Disponível em: http://www.who.int/ageing/ projects/5.Intervention, $\% 20$ policies $\% 20$ and $\% 20$ sustainability $\% 20$ of $\% 20$ falls $\% 20$ prevention.pdf. Background Paper: Falls Prevention: Policy, Research and Practice

9. Coimbra AMV, Ricci NA, Coimbra IB, Costallat LTL. Falls in the elderly of the family health program. Arch Gerontol Geriatr 2010;51(3):317-22.

10. Siqueira FV, Facchini LA, Silveira DS, Piccini RX, Tomasi E, Thumé E et al. Prevalência de quedas em idosos no Brasil: uma análise nacional. Cad Saúde Pública 2011;27(9):1819-26.

11. Milat JÁ, Watson Wl, Monger C, Barr M, Giffin M, Reid M. Prevalence, circumstances and consequences of falls among community-dwelling older people: results of the 2009 NSW Falls Prevention Baseline Survey. N. S. W. Public Health Bull 2011;22(3-4):438.

12. American Geriatrics Society; British Geriatrics Society. Summary of the updated American Geriatrics Society/British Geriatrics Society clinical practice guideline for prevention of falls in older persons. J Am Geriatr Soc 2011;59:148-57. 
13. Portal da saúde [Internet]. Brasília: Ministério da Saúde. 2011. Quedas de idosos; [acesso em 02 out 2012]. Disponível em:http://portal. saude.gov.br/portal/saude/visualizar_texto. cfm?idtxt $=33674 \&$ janela $=1$

14. Associação Médica Brasileira; Conselho Federal de Medicina. Projeto Diretriz. Brasília: AMB: CFM; 1999.

15. Ciaschini PM, Straus SE, Dolovich LR, Goeree RA, Leung KM, Woods CR et al. Community-based intervention to optimise falls risk management: a randomised controlled trial. Age Ageing 2009;38(6):724-30.

16. Al-Aama T. Falls in the elderly: spectrum and prevention. Can Fam Physician 2011;57(7): 771-6.

17. Nicolussi AC, Fhon JRS, Santos CAV, Kusumota L, Marques S, Rodrigues RAP. Quality of life in elderly people that have suffered falls: integrative literature review. Ciênc Saúde Coletiva 2012;17(3):723-30.

18. Czerwinski E, Białoszewski D, Borowy P, Kumorek A, Białoszewski A. Epidemiology, clinical significance, costs and fall prevention in elderly people. Ortop Traumatol Rehabil 2008;10(5):419-28.

19. Coutinho ESF, Bloch KV, Rodrigues LC. Characteristics and circumstances of falls leading to severe fractures in elderly people in Rio de Janeiro, Brazil. Cad Saúde Pública 2009;25(2):455-9.

20. Morley JE, Rolland Y, Tolson D, Vellas B. Increasing awareness of the factors producing falls: the mini falls assessment [Editorial]. J Am Med Dir Assoc 2012;13(2):87-90.

21. Bleijlevens MHC, Diederiks JPM, Hendriks MRC, Van Haastregt JCM, Crebolder HFJM, Van Eijk JTHM. Relationship between location and activity in injurious falls: an exploratory study. BMC Geriatr 2010;10:1-9.

22. Kwan MM, Close JCT, Wong AKW, Lord SR. Falls incidence, risk factors, and consequences in chinese older people: a systematic review. J Am Geriatr Soc 2011;59:536-43.

23. Organização Mundial da Saúde. Relatório global da OMS sobre prevenção de quedas na velhice. De Campos LM, tradutora. São Paulo: Secretaria de Estado da Saúde, 2010.

24. Scheffer AC, Schuurmans MJ, Dijk NV, Hooft TV, Rooij SE. Fear of falling: measurement strategy, prevalence, risk factors and consequences among older persons. Age Ageing 2008;37(1):19-24.

25. Fabre JM, Ellis R, Kosma M, Wood RH. Falls risk factors and a compendium of falls risk screening instruments. J Geriatr Phys Ther 2010;33:184-97.
26. Peeters G, Schoor NM, Lips P. Fall risk: the clinical relevance of falls and how to integrate fall risk with fracture risk. Best Pract Res, Clin Rheumatol 2009;23(6):797-804.

27. Gschwinda YJ, Wolfa I, Bridenbaugha SA, Kressig RW. Basis for a Swiss perspective on fall prevention in vulnerable older people. Swiss Med Wkly 2011;141:1-14.

28. Almeida ST, Soldera CLC, Carli GA, Gomes I, Resende L. Análise de fatores extrínsecos e intrínsecos que predispõem a quedas em idosos. Rev Assoc Med Bras 2012;58(4):427-33.

29. Fox PJ, Vazquez L, Tonner C, Stevens JA, Fineman N, Ross LK. A randomized trial of a multifaceted intervention to reduce falls among communitydwelling adults. Health Educ Behav 2010;37(6):831-48.

30. Stevens JA, Baldwin GT, Ballesteros MF, Noonan RK, Sleet DA. An older adult falls research agenda from a public health perspective. Clin Geriatr Med 2010;26(4):767-79.

31. Galizia G, Langellotto A, Cacciatore F, Mazzella F, Testa G, Della-Morte D et al. Association between nocturia and falls-related long-term mortality risk in the elderly. J AM Med Dir Assoc 2012;13(7):640-44.

32. Dias RC, Freire MTF, Santos EGS, Vieira RA, Dias JMD, Perracini, MR. Características associadas à restrição de atividades por medo de cair em idosos comunitários. Rev Bras Fisioter 2011;15(5):406-13.

33. Tinetti ME, Kumar C. The patient who falls: "it's always a trade-off.” J Am Med Assoc 2010;303(3):258-66.

34. Deandrea S, Lucenteforte E, Bravi F, Foschi R, La Vecchia C, Negri E. Risk factors for falls in community-dwelling older people: a systematic review and meta-analysis. Epidemiology 2010;21(5):658-68.

35. Ricci NA, Gonçalves DFF, Coimbra AMV, Coimbra IB. Sensory interaction on static balance: a comparison concerning the history of falls of community-dwelling elderly. Geriatr Gerontol Int 2009;9(2):165-71

36. Michael YL, Whitlock EP, Lin JS, Fu R, O'Connor EA, Gold, R. Primary Care-Relevant Interventions to Prevent Falling in Older Adults: a systematic evidence review for the U.S. Preventive Services Task Force. Ann Intern Med 2010;153(12):815-25.

37. Elsawy B, Higgins KE. The Geriatric Assessment. Am Fam Physician 2011; 83(1):48-56.

38. Gates S, Lamb SE, Fisher JD, Cooke MW, Carter YH. Multifactorial assessment and targeted intervention for preventing falls and injuries among older people in community and emergency care settings: systematic review and meta-analysis. Br Med J 2008;336(7636):130-33. 
39. Balzer K, Bremer M, Schramm S, Lühmann D, Raspe H. Falls prevention for the elderly. GMS Health Technol Assess 2012;8:1-18.

40. Freiberger E, Haberle L, Spirduso EE, Zijlstra GAR. Long-term effects of three multicomponent exercise interventions on physical performance and fall-related psychological outcomes in community-dwelling older adults: a randomized controlled trial. J Am Geriatr Soc 2012;60(3):437-46.

41. Choi M, Hector M. Effectiveness of intervention programs in preventing falls: a systematic review of recent 10 years and meta-analysis. J Am Med Dir Assoc 2012;13(2):13-21.

Recebido: 04/4/2013

Revisado: 06/4/2014

Aprovado: 15/7/2014 